\title{
Evolutionary upgrade of stefins for secretion in parasites
}

\section{Michal Buša ${ }^{1}$, Zuzana Matoušková ${ }^{1}$, Pavlína Řezáčová1, Pavla Sojková-Bartošová ${ }^{2}$, Martin Horn ${ }^{1}$, Saša Štefanič ${ }^{3}$, Michael Mareš ${ }^{1}$}

${ }^{1}$ Institute of Organic Chemistry and Biochemistry, Czech Academy of Sciences, Flemingovo nám. 2, Prague, 166 10, Czechia;

${ }^{2}$ Institute of Parasitology, Biology Centre of Czech Academy of Sciences, Branišovská 1160/31, České Budějovice, 370 05, Czechia; ${ }^{3}$ Institute of Parasitology, University of Zurich, Winterthurerstrasse, Zurich, CH-8057, Switzerland;

\section{busa@uochb.cas.cz}

Fasciolosis caused by the liver fluke Fasciola hepatica is a worldwide spread parasitic disease of ruminant and an emerging human disease. Cystatin superfamily of cysteine protease inhibitors is composed of intracellular type 1 cystatins (stefins), secreted type 2 cystatins, and multidomain type 2 cystatins. Helminth parasites secrete type 2 cystatins to modulate host immune responses for successful parasitism, except for F. hepatica that lacks type 2 cystatin genes.

This work is focused on F. hepatica type 1 cystatin FhCY2. It was localized to gastroderm and tegument and was surprisingly detected in the excretory/secretory products. We demonstrated that recombinant FhCY2 is a broad-selective inhibitor of host cysteine cathepsins as well as cysteine cathepsins of F. hepatica, suggesting its dual role in the regulation of exogenous and endogenous proteolytic systems. Furthermore, we solved the crystal structure of FhCY2 at $1.6 \AA$. The structural and phylogenetic analyses revealed that FhCY2 has the sequence and fold of type 1 cystatins but also the signal peptide and disulfides typical for type 2 cystatins, combining all hallmarks in an unprecedented way. We propose that FhCY2 is an evolutionary upgrade of type 1 cystatins for secretion that occurred in F. hepatica (and Fasciolidae family in general) in the absence of type 2 cystatins.

Keywords: stefin, cystatin, protease inhibition, protein crystallography, cathepsin 